\title{
A possibility of magnetic field biasing tunable inductive device using a hard magnetic film magnetized by pulsed-magnetic field
}

\author{
Yosuke Obinata, Megumi Yuki, Makoto Sonehara, Member, IEEE, Yutaka Kuramoto, Kunihiko Suzuki, \\ and Toshiro Sato, Member, IEEE
}

Spin Device Technology Center, Shinshu University, 4-17-1 Wakasato, Nagano 380-8553, Japan

\begin{abstract}
In this paper, the authors have proposed a possibility of a magnetic field biasing tunable inductive device. To confirm a new scheme, a tunable coplanar waveguide (CPW) resonator with a combination of soft magnetic thin film and hard magnetic film has been fabricated and evaluated. The magnetic pole of hard magnetic film can be controlled by magnetization using a pulsed magnetic field. A bias magnetic field is applied in the soft magnetic film from the magnetic pole of the hard magnet film. Consequently, permeability of the soft magnetic film can be controlled by changing amplitude of the pulsed magnetic field in the magnetizing for the hard magnetic film. A $20 \mathrm{~mm}$ long coplanar wave guide resonator has been fabricated using $\mathrm{FeSiO} / \mathrm{SiO}_{2}$ granular multilayer film and $\mathrm{FeCoSm}$ amorphous hard magnetic film. From the experimental results, in case of using $0.2 \mu \mathrm{m}$ thick soft granular film, by changing amplitude of the $1 \mathrm{~ms}$ width currentpulse for magnetizing pulsed magnetic field for hard magnetic film, the maximum inductance change was up to $18 \%$, and maximum change of the resonant frequency was $9.6 \%$. The control energy for one time tuning was small enough $(5.4 \mu \mathrm{Wh})$.
\end{abstract}

Index Terms - Tunable inductive device, cell phone, hard magnetic film, soft magnetic film, pulsed-current magnetization method.

\section{INTRODUCTION}

$\mathrm{T}$ he cell phone with built-in various functions such as GPS, wireless LAN, and terrestrial digital television broadcast has been developed and spread. In addition, the call function with multi-band has been required for the international roaming. Such multi-band service requires many $\mathrm{RF}$ circuits, for example, $L C$-VCO ( $L C$ tank voltagecontrolled oscillator) and impedance matching element are necessary for each of multi-band frequencies. Since many RF passive devices such as inductor and capacitor are used in the multi-band RF front-end circuits, the development of the tunable RF passives is very important issue to realize small RF front-end circuit.

Many researchers have reported the tunable RF inductive devices. For example, K. Okada et al. [1] reported a MEMS tunable air-core inductor with a moving metal plate. This had a very complex structure because of a set-up of the MEMS actuator, and the maximum quality factor was low owing to the eddy current in the moving metal plate. On the other hand, B. K. Kuanr et al. [2] reported a tunable FMR (ferromagnetic resonance) filter using bias magnetic field applied in easy magnetization direction for the magnetic film. The control power for changing FMR frequency becomes large in case of using dc current for generating bias magnetic field. In addition, in order to maintain the fixed FMR frequency, the dc current must be kept a constant value. Moreover, the multiferroic (ferromagnetic/piezoelectric composite) materials for voltagecontrolled tunable inductive devices have been investigated [3]-[5]. For example, G. Srinivasan [3] reviewed a composite of magnetostrictive/piezoelectric phases and their possible great applications such as magnetic field sensors, electric field

Manuscript received Xxxxx XX, 2012; revised October 31, 2012. Current version published Xxxxx XX, 2012. Corresponding author: Makoto Sonehara (e-mail: makoto@shinshu-u.ac.jp; optional phone: +81-26-269-5198; optional fax: +81-26-269-5215)

Digital Object Identifier 10.1109/TMAG.2012.xxxxxxx tunable microwave/millimeter-wave devices, and miniature antennas. Such voltage-controlled tunable device has a great advantage of negligible small control power.

Above mentioned conventional tunable devices have no self-holding function, because the control voltage or current must be kept to constant value in order to maintain a fixed magnetic property such as permeability and FMR frequency.

In this study, the authors have proposed a magnetic field biasing tunable RF soft magnetic device, which has a combination of the soft and hard magnetic film. The magnetic pole of the hard magnetic film can be changed using magnetization controlled by a pulsed magnetic field. Bias magnetic field is applied in the soft magnetic film from magnetic pole of the hard magnetic film. Hence, the permeability of the soft magnetic film can be controlled by changing amplitude of the pulsed magnetic field, and after magnetization it can be maintained without the control power.

This paper describes a possibility of the proposed method for tunable inductive device. A coplanar waveguide (CPW) resonator with granular soft magnetic film and amorphous hard magnetic film has been demonstrated.

\section{BASIC PRINCIPLE OF TUNABLE PERMEABILITY OF SOFT MAGNETIC FILM}

Fig 1, 2 and 3 show a basic principle of the permeability control for the soft magnetic film using bias magnetic field of hard magnetic film. Fig. 1 shows a schematic explanation of control method for hard axis permeability of soft magnetic film. As shown in Fig. 2, the hard magnetic film is magnetized by a pulsed-magnetic field generated by pulsed-current flowing in the magnetizing coil. The pulsed-current consists of a negative current pulse with constant amplitude $I_{\mathrm{p} \text { - and a }}$ positive current pulse with variable amplitude $I_{\mathrm{p}^{+}}$to change the remanent magnetization $M_{\mathrm{r}}$ of the hard magnetic film.

As shown in Fig. 1, the bias magnetic field $H_{\mathrm{DC}}$ is applied in easy axis of soft film, which can be controlled by changing 


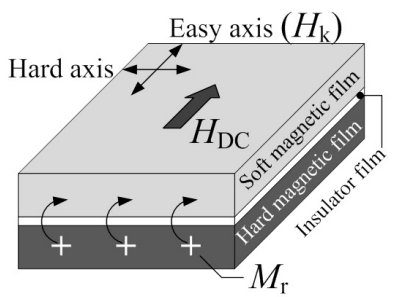

Fig. 1. Schematic explanation of control method for hard axis permeability of soft magnetic film by bias magnetic field using hard magnetic film.
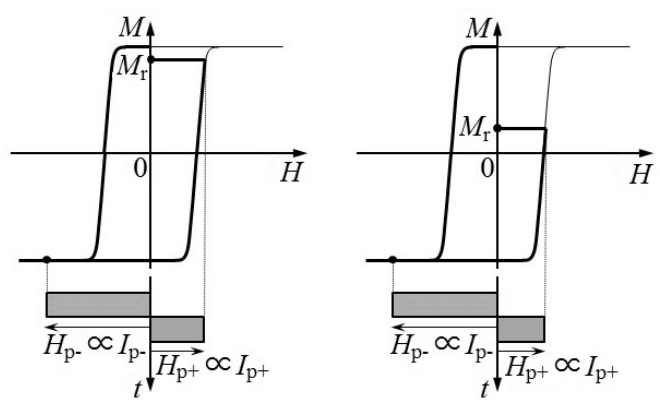

Fig. 2. Magnetization controlled by a pulsed-magnetic field for hard magnetic film.

$M_{\mathrm{r}}$ of the hard magnetic film. The hard axis relative permeability $\mu_{\mathrm{r}}$ of soft magnetic film can be expressed in Eq. 1 and can be changed as shown in Fig. 3;

$$
\mu_{\mathrm{r}}=\frac{M_{\mathrm{s}}}{\mu_{0}\left(H_{\mathrm{k}}+\left|H_{\mathrm{DC}}\right|\right)}+1 \cong \chi_{\mathrm{r} 0} \cdot \frac{1}{1+\frac{\left|H_{\mathrm{DC}}\right|}{H_{\mathrm{k}}}}
$$

where $M_{\mathrm{s}}$ is the saturation magnetization, $\mu_{0}$ is the permeability in vacuum, $H_{\mathrm{k}}$ is the intrinsic uniaxial anisotropy field, and $\chi_{\mathrm{r} 0}$ is the hard axis relative susceptibility when $H_{\mathrm{DC}}$ $=0$. Hard axis permeability is inversely proportional to the bias magnetic field.

\section{EXPERIMENT}

\section{A. Structure of coplanar waveguide resonator}

To confirm a possibility of the tunable inductive device, a coplanar waveguide (CPW) resonator has been fabricated.

Fig. 4 shows a structure of the fabricated CPW shortterminated stub with top and bottom magnetic film layer consisting of soft magnetic $\mathrm{FeSiO} / \mathrm{SiO}_{2}$ granular multilayer film and $\mathrm{FeCoSm}$ amorphous hard magnetic film. FeSiO granular film had an intrinsic FMR frequency around 900 $\mathrm{MHz}$ and wide FMR linewidth [6], therefore a $20 \mathrm{~mm}$ long CPW was designed for operating at several hundred megahertz. Straight signal line consisted of $3 \mu \mathrm{m}$ thick, $50 \mu \mathrm{m}$ wide $\mathrm{Cu}$ conductor with $50 \mathrm{~nm}$ thick $\mathrm{Cr}$ underlayer, which was sandwiched by top and bottom magnetic layer.

The fabricated short-terminated CPW stub functions as a resonator when a quarter wavelength of signal propagation is equal to line-length at a $L C$ resonant frequency (quarter wavelength frequency $f_{\lambda / 4}$ ). As shown in Fig. $4(\mathrm{~d})$, since the line-inductance can be enhanced by inner soft magnetic $\mathrm{FeSiO} / \mathrm{SiO}_{2}$ magnetic films, it can be changed by tunable permeability based on the hard magnetic film magnetized by

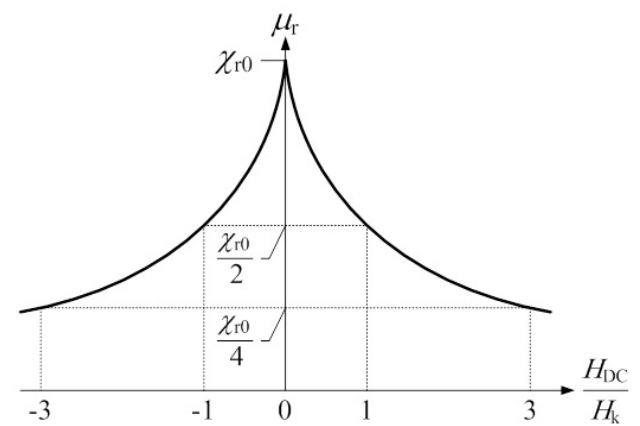

Fig. 3. Change in relative permeability due to bias magnetic field by hard magnetic film.

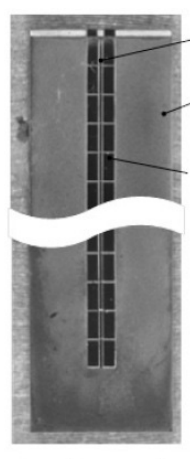

(a) Photograph

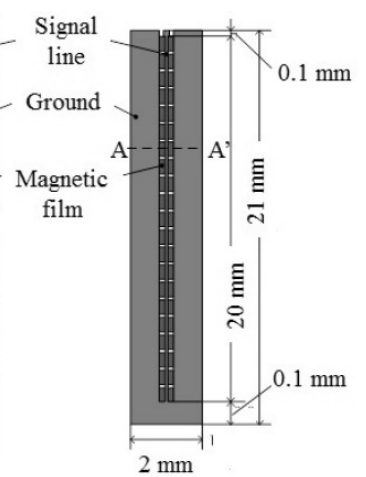

(b) Top schematic view

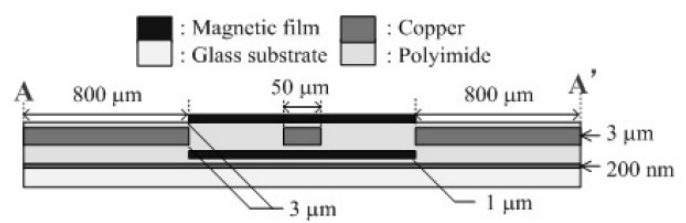

(c) A-A' cross-section

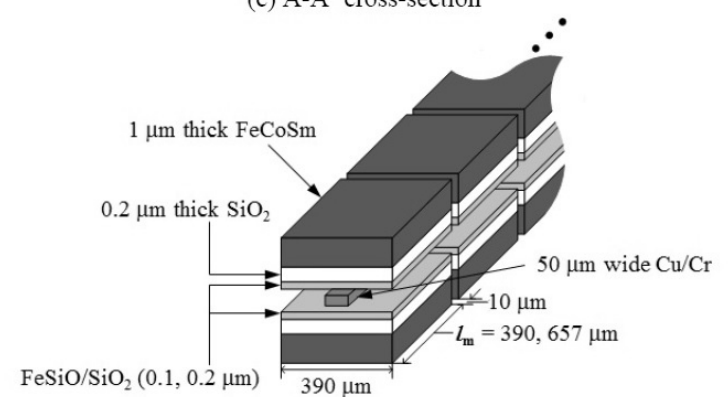

(d) Arrangement of a top and bottom magnetic layer consisting of $\mathrm{FeSiO} / \mathrm{SiO}_{2}$ granular multilayer film and $\mathrm{FeCOSm}$ amorphous hard magnetic film

Fig. 4. Structure and dimensions of coplanar waveguide resonator fabricated.

pulsed-magnetic field, and resonant frequency can be changed.

To improve the spatial distribution of bias magnetic field in the soft magnetic film, a divided magnetic film structure composed of short magnetic film segments and air-gap has been introduced [6]. In the fabricated CPW, two different segment length $l_{\mathrm{m}}$ of 390 and $657 \mu \mathrm{m}, 10 \mu \mathrm{m}$ width air-gap have been introduced, as shown in Fig. 4(d).

\section{B. Fabrication of magnetic film}

Magnetic film for CPW resonator was fabricated by inductively coupled RF sputtering system under Ar gas 


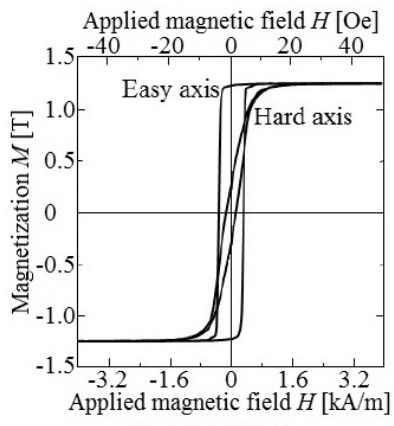

(a) $\mathrm{FeSiO} / \mathrm{SiO}_{2}$

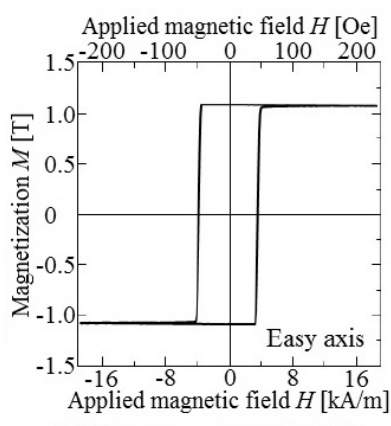

(b) $\mathrm{Fe}_{27.5} \mathrm{Co}_{66.8} \mathrm{Sm}_{5.7}$ (at.\%)

Fig. 5. Magnetization curves of $\mathrm{FeSiO} / \mathrm{SiO}_{2}$ and $\mathrm{FeCoSm}$ film.

Table 1 Three types of magnetic films used in the fabricated CPW.

\begin{tabular}{c|c|c|c}
\hline Sample name & $\mathrm{S} 1$ & $\mathrm{~S} 2$ & $\mathrm{~L} 2$ \\
\hline Total FeSiO thickness $t_{\mathrm{s}}$ & $0.1 \mu \mathrm{m}$ & \multicolumn{2}{|c}{$0.2 \mu \mathrm{m}$} \\
\hline $\begin{array}{c}\text { Segment length } l_{\mathrm{m}} \text { in magnetic } \\
\text { film with divided structure }\end{array}$ & \multicolumn{2}{|c|}{$390 \mu \mathrm{m}$} & $657 \mu \mathrm{m}$ \\
\hline
\end{tabular}

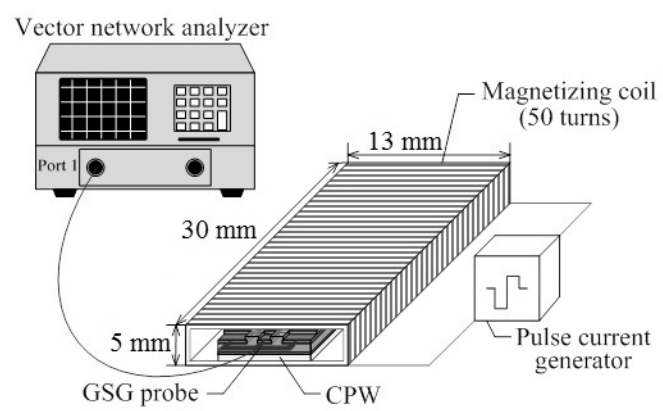

Fig. 6. Experimental set-up for evaluation of the fabricated coplanar waveguide resonator. 150-turn-magnetizing coil has a resistance of $3.4 \Omega$.

pressure of $0.22 \mathrm{~Pa}$ at room temperature. Static magnetic field $(8 \mathrm{kA} / \mathrm{m})$ was applied in the film plane during deposition in order to introduce in-plane uniaxial magnetic anisotropy.

$\mathrm{FeSiO} / \mathrm{SiO}_{2}$ granular multilayer soft magnetic film was deposited by co-sputtering using $\mathrm{Fe}$ and $\mathrm{SiO}_{2}$ targets. Each $\mathrm{FeSiO}$ granular layer thickness was $6 \mathrm{~nm}$ and $\mathrm{SiO}_{2}$ layer thickness was $1 \mathrm{~nm}$. Two types of the magnetic film with 0.1 and $0.2 \mu \mathrm{m}$ total thickness of $\mathrm{FeSiO}$ granular layers were used for CPW. $1 \mu \mathrm{m}$ thick FeCoSm amorphous hard magnetic film was deposited by co-sputtering using $\mathrm{Fe}$, Co and $\mathrm{Sm}$ targets, the deposited film composition was $\mathrm{Fe}_{27.5} \mathrm{Co}_{66.8} \mathrm{Sm}_{5.7}$ (at.\%).

The magnetization curves of $\mathrm{FeSiO} / \mathrm{SiO}_{2}$ and $\mathrm{FeCoSm}$ film are shown in Fig. 5. Uniaxial anisotropy magnetic field of $\mathrm{FeSiO} / \mathrm{SiO}_{2}$ film was about $0.8 \mathrm{kA} / \mathrm{m}$.

Very large coercive force of the hard magnetic film contributes to the stable remanent-state, however very large pulsed magnetic field is required for re-magnetizing. The deposited FeCoSm film had an easy axis coercive force of 4 $\mathrm{kA} / \mathrm{m}$, which was not so large and it is easy to re-magnetize using reasonable strength pulsed magnetic field. Unstable tunable device property owing to the small coercive force of hard magnetic film can be compensated by using refreshing pulsed magnetic field magnetization.

FeCoSm film had a very large uniaxial anisotropy magnetic field of $160 \mathrm{kA} / \mathrm{m}$ (not shown in Fig. 5) and very low hard-

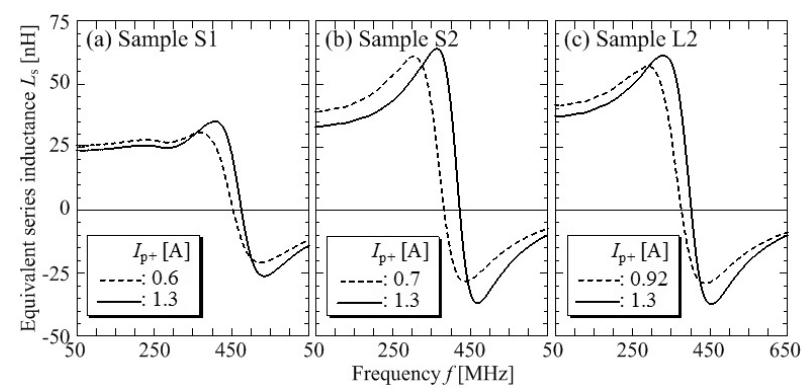

Fig. 7. Frequency dependence of equivalent series inductance $L_{\mathrm{s}}$ in three kinds of CPWs, sample S1, S2 and L2.

axis susceptibility. Therefore a contribution of FeCoSm film to the line-inductance enhancement in the $\mathrm{CPW}$ is considered to be very small.

Three types of magnetic films were used in the fabricated CPW, sample S1, S2 and L2 are shown in Table 1.

\section{Experimental set-up for $C P W$ resonator fabricated}

The fabricated CPW has been evaluated using an experimental set-up with external magnetizing coil, as shown in Fig. 6. Equivalent series inductance $L_{\mathrm{s}}$ was obtained using following equations;

$$
L_{\mathrm{s}}=\frac{X_{\mathrm{s}}}{\omega}=\frac{\operatorname{Im}\left[Z_{\mathrm{s}}\right]}{\omega}=\omega^{-1} \operatorname{Im}\left[\frac{1+S_{11}}{1-S_{11}} Z_{0}\right]
$$

where $X_{\mathrm{s}}$ is the equivalent series reactance of the CPW, $\omega$ is the angular frequency, $Z_{\mathrm{s}}$ is the input impedance, $S_{11}$ is the reflection coefficient measured using a vector network analyzer (Hewlett Packard; 8720D), and $Z_{0}$ is $50 \Omega$.

The pulsed-current with negative and positive current pulse $I_{\mathrm{p}-}, I_{\mathrm{p}+}$ were generated by pulse current generator. In this experiment, current pulse-width was $1 \mathrm{~ms}$. The negative current

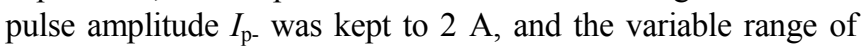
the positive current pulse amplitude $I_{\mathrm{p}^{+}}$was 0.2 to $1.3 \mathrm{~A}$.

\section{RESULTS AND DISCUSSION}

Fig. 7 shows the frequency dependence of the equivalent series inductance $L_{\mathrm{s}}$ in the three kinds of the CPWs, sample S1, $\mathrm{S} 2$ and L2. From Fig. 7, the equivalent series inductance $L_{\mathrm{s}}$ and resonant frequency $f_{\lambda / 4}$ can be controlled by changing the positive current-pulse $I_{\mathrm{p}+}$.

Fig. 8 shows the relation between $L_{\mathrm{s}}, f_{\lambda / 4}$ and $I_{\mathrm{p}+}$. The positive peak magnetizing field $H_{\mathrm{p}^{+}}$for FeCoSm hard magnetic film is also indicated in Fig. 8. Table 2 shows the summarized the maximum change in $L_{\mathrm{s}}$ at $50 \mathrm{MHz}$ and $f_{\lambda / 4}$.

From the experimental results of Sample S1 and S2, when increasing soft magnetic layer thickness, both the changes in $L_{\mathrm{s}}$ and $f_{\lambda / 4}$ became large. However, the maximum change of inductance was below $20 \%$ even in Sample S2. Effective permeability $\mu_{\text {eff. }}$ on the inductance enhancement in the CPW is defined as follows;

$$
\mu_{\text {eff. }}=L_{\mathrm{s}} / L_{\mathrm{o}}
$$

where $L_{\mathrm{o}}$ is the air-core inductance. Inductance enhancement $L_{\mathrm{s}} / L_{\mathrm{o}}$ depends strongly on the magnetic layer thickness. Since the magnetic layer thickness is relatively small $(0.2 \mu \mathrm{m}$ thick 


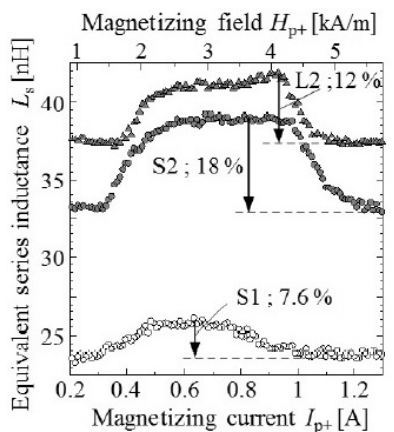

(a) $L_{\mathrm{s}}$ vs. $I_{\mathrm{p}^{+}}$

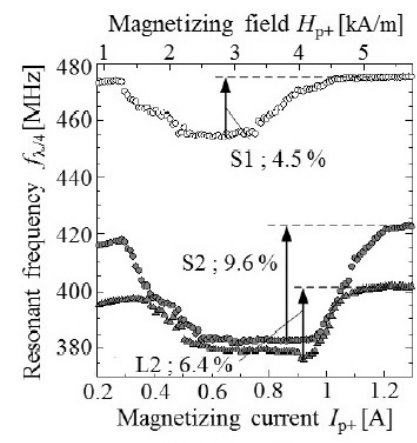

(b) $f_{\lambda / 4}$ vs. $I_{\mathrm{p}^{+}}$
Fig. 8. Relation between equivalent series inductance $L_{\mathrm{s}}$, resonant frequency $f_{\lambda / 4}$ and amplitude of positive magnetizing current-pulse $I_{\mathrm{p}+}$.

Table 2 Maximum change in equivalent series inductance $L_{\mathrm{s}}$, maximum change in resonant frequency $f_{\lambda / 4}$ in three kinds of CPWs.

\begin{tabular}{c|c|c|c}
\hline Sample name & $\mathrm{S} 1$ & $\mathrm{~S} 2$ & $\mathrm{~L} 2$ \\
\hline $\begin{array}{c}\text { Maximum change in inductance } \\
L_{\mathrm{S}}(\text { at } 50 \mathrm{MHz})\end{array}$ & $7.6 \%$ & $18 \%$ & $12 \%$ \\
\hline $\begin{array}{c}\text { Maximum change in quarter } \\
\text { wavelength frequency } f_{\lambda / 4}\end{array}$ & $4.5 \%$ & $9.6 \%$ & $6.4 \%$ \\
\hline
\end{tabular}

FeSiO in Sample S2), the effective permeability $\mu_{\text {eff. }}$ becomes much smaller than intrinsic permeability of the $\mathrm{FeSiO}$ magnetic film. To obtain wider tunable inductance, higher effective permeability due to thicker magnetic core should be introduced.

FeCoSm film had very large uniaxial magnetic anisotropy of $160 \mathrm{kA} / \mathrm{m}$ and very low hard-axis permeability. Therefore a contribution to the line-inductance enhancement is very week, and additional loss owing to the FeCoSm filmt is considered to be negligible small. However, the quality factor, measured at frequencies lower than $L C$ resonant frequency $f_{\lambda / 4}$, was not so high (below 10), because the FMR loss of $\mathrm{FeSiO} / \mathrm{SiO}_{2}$ soft magnetic film was still large even in the lower frequencies than FMR, which was owing to the wide FMR linewidth.

From the experimental results of Sample S2 and L2, when decreasing the segment length $l_{\mathrm{m}}$ in the divided magnetic film structure, both the change in $L_{\mathrm{s}}$ and $f_{\lambda / 4}$ became large. Bias magnetic field applied in the soft magnetic film decreases with increasing magnetic segment length $l_{\mathrm{m}}$ in the divided magnetic film structure, because the long distance between magnetic poles at both ends of each magnetic segment degrades bias magnetic field strength.

Since the proposed method has the self-holding function, tunable operation toward a target value requires only one time magnetizing process for hard magnetic film. By using the parameters of the fabricated tunable $\mathrm{CPW}$, the control energy $E_{\mathrm{c}}$ for one time tunable operation was estimated using the following calculation;

$$
E_{\mathrm{c}}=\left(I_{\mathrm{p}-}{ }^{2} T_{-}+I_{\mathrm{p}+}{ }^{2} T_{+}\right) R_{\mathrm{m}}
$$

In the experiments, magnetizing pulsed-current had a negative current-pulse amplitude $I_{\mathrm{p} \text { - }}$ of $2 \mathrm{~A}$, positive current-pulse amplitude $I_{\mathrm{p}-}$ of $1.3 \mathrm{~A}$ maximum, and both current-pulse width $T$. and $T_{+}$of $1 \mathrm{~ms}$. Since the magnetizing coil resistance $R_{\mathrm{m}}$ was $3.4 \Omega$, the maximum control energy $E_{\mathrm{c}}$ for one time tuning was estimated to be about $19.3 \mathrm{~mJ}(5.4 \mu \mathrm{Wh})$. Typical energy of the Li-ion battery used in the cell phones is about 3 Wh. Hence it is considered that the control energy for one time tuning is small enough when comparing with that of the battery.

Although the control energy may be larger than that of voltage controlled multiferroic based tunable device, the proposed scheme has a specific feature of "self-holding function" based on the hard magnetic film.

\section{CONCLUSIONS}

A tunable inductive device with a combination of soft magnetic thin film and hard magnetic film has been proposed. In order to confirm a possibility of a magnetic field biasing tunable device controlled by pulsed-magnetic field magnetizing method, a $20 \mathrm{~mm}$ long coplanar wave guide resonator with soft magnetic $\mathrm{FeSiO} / \mathrm{SiO}_{2}$ granular multilayer film and $\mathrm{FeCoSm}$ amorphous hard magnetic film was fabricated and evaluated. From the experimental results, in case of using $0.2 \mu \mathrm{m}$ thick soft granular film, by changing amplitude of $1 \mathrm{~ms}$ width pulse current for pulsed magnetic field applied in the hard magnetic film, the maximum inductance change of $18 \%$ was obtained, and the maximum resonant-frequency change of $9.6 \%$ was obtained. The control energy for one time tuning was small enough (5.4 $\mu \mathrm{Wh}$ ) compared with energy of the Li-ion battery used in cell phones.

The conventional tunable devices have no "self-holding function", because the control voltage or current must be kept to constant value in order to maintain a fixed magnetic property. Since the new scheme proposed here is hard magnet based device, after pulsed-magnetization the device property can be maintained without the control signal. Such selfholding function will be very useful to realize the simple control circuit.

\section{REFERENCES}

[1] K. Okada, H. Sugawara, and K. Masu, "Reconfigurable RF CMOS Circuit Using On-Chip MEMS Variable Inductor", IEICE Tech. Report, Vol.105, No.258, pp.45-50 (2005). (in Japanese)

[2] B. K. Kuanr, V. Veerakumar, K. Lingam, S.R. Mishra, Alka V.Kuanr, R.E. Camley, and Z. Celinski, "Microstrip-Tunable Band-Pass Filter Using Ferrite (Nanoparticles) Coupled Lines", IEEE Trans. Magn., Vol.45, No.10, pp.4226-4229 (2009).

[3] G. Srinivasan, "Magnetoelectric Composites", Annual Review of Mater. Research, Vol.40, pp. 153-178, (2010).

[4] Y. Chen, A. Daigle, T. Fitchorov, B. Hu, M. Geiler, A. Geiler, C. Vittoria, V. G.Harris, "Electronic tuning of magnetic permeability in $\mathrm{Co}(2) \mathrm{Z}$ hexaferrite toward high frequency electromagnetic device miniaturization", Applied Physics Lett., Vol. 98, No.20, (2011).

[5] S. Sheng, C. K. Ong, "Multifunctional dual-tunable multiferroic $\mathrm{Ba}_{0.25} \mathrm{Sr}_{0.75} \mathrm{TiO}_{3}-\mathrm{BiFeO}_{3}-\mathrm{Ba}_{0.25} \mathrm{Sr}_{0.75} \mathrm{TiO}_{3}$ trilayered structure for tunable microwave applications", Jour. of Physics D-Appl. Physics, Vol.44, No.16, (2011).

[6] M. Yuki, M. Sonehara, T. Sato, and K. Ikeda, "Fundamental Study on Tunable RF Magnetic Devices Using DC Bias Magnetic Field Generated by Permanent Magnet Film", J. Magn. Soc. Jpn., Vol.36, No.3, pp.229234 (2012). (in Japanese) 